\title{
Processos de Soldagem Sustentáveis - Estudo de Caso de Aplicação de Revestimento Duro em Moendas de Cana de Açúcar
}

\author{
Marcio de Queiroz Murad ${ }^{1}$, Valtair Antônio Feraressi², Wisley Falco Sales ${ }^{1}$ (D) \\ ${ }^{1}$ Universidade Federal do Triângulo Mineiro - UFTM, Uberaba, MG, Brasil. \\ ${ }^{2}$ Universidade Federal de Uberlândia - UFU, Uberlândia, MG, Brasil.
}

Como citar: Murad MQ, Feraressi VA, Sales WF. Processos de soldagem sustentáveis - estudo de caso de aplicação de revestimento duro em moendas de cana de açúcar. Soldagem \& Inspeção. 2020;25:e2532. https://doi.org/10.1590/0104-9224/SI25.32

\begin{abstract}
Resumo: A avaliação da sustentabilidade dos processos de soldagem é focada principalmente na geração de resíduos. Dentro desta linha de pesquisa, quando se analisa os respingos provenientes da soldagem, como um resíduo contaminante e uma perda de recursos financeiros, percebe-se que este assunto é pouco explorado. Assim, este trabalho apresenta uma análise relacionada a sustentabilidade na aplicação de revestimento duro pelo processo FCAW. O objetivo é estudar a influência do parâmetro velocidade de aplicação de revestimento (VC) no Rendimento de Deposição (Rd) aplicado ao revestimento duro em camisas de moendas. Os aspectos relacionados a Triple Bottom Line foram discutidos em pró de uma manufatura sustentável e serão focados nos pilares econômicos e ambientais neste estudo. Como ponto de partida, entrou-se em contato como uma empresa parceira que executa esse procedimento utilizando Vc $10 \mathrm{~m} / \mathrm{min}$. Como proposta este estudo, utilizando Vc de 6, 10 e 13,6 m/min foi calculado o Rd para as 3 situações. Os resultados mostraram uma melhora no Rd quando se reduziu a Vc. Esta melhoria reflete em uma economia de aproximadamente $\mathrm{R} \$ 6.7$ milhões por ano e se evitaria de gerar 385 toneladas de materiais perigosos no Brasil. Resultados estes que contribuem para uma produção mais sustentável.
\end{abstract}

Palavras-chave: Sustentabilidade; Manufatura sustentável; Soldagem; Rendimento de deposição; Moendas.

\section{Sustainable Welding Processes - Case Study of Hard Coating Application in Sugar Cane Mills}

\begin{abstract}
Studies that are carried out in the evaluation of the sustainability of welding processes, are mainly focused on waste generation issues. Within this line of research, when analyzing spatter from welding, as a contaminating residue and a loss of financial resources, it is clear that this subject is little explored. Thus, this work presents an analysis on the application of hard coating by the Flux-Cored Arc Welding (FCAW) process. The objective is to study the influence of the welding speed parameter on the deposition yield in the application of hard coating, in milling liners. The aspects related to the Triple Bottom Line were discussed in favor of sustainable manufacturing and will be focused on the economic and environmental pillars in this study. As a starting point, we contacted a partner company that performs this procedure using peripheral welding speeds with $10 \mathrm{~m} / \mathrm{min}$. As proposed in this study, using speeds of 6 and $13.6 \mathrm{~m} / \mathrm{min}$, the deposition yield for the 3 speeds was calculated. There was an 20,22\% improvement in yield when the speed was reduced from 10 to $6 \mathrm{~m} / \mathrm{min}$. In the sugar and alcohol industries in Brazil, this improvement is reflected in savings of U\$ 1,4 million per year and would avoid generating 385,000 kg of hazardous materials.
\end{abstract}

Key-words: Sustainability; Sustainable manufacturing; Welding; Deposition yield; Mills.

\section{Introdução}

As situações de chão de fábrica necessitam de uma especificação de soldagem qualificada para obter qualidade no produto final [1]. Em situações práticas, ensaios podem ser realizados com o objetivo de definir os parâmetros mais adequados para uma aplicação específica, oferecendo com isso a possibilidade de rastrear as soluções que elevam a eficiência do processo [1,2].

De um modo geral os processos de soldagem são muito comuns nas indústrias, devido a sua larga aplicação, porém alguns resultados não esperados provocam impactos ambientais negativos, como exemplo a emissões, geração de resíduos e utilização ineficiente de energia e materiais [1].

A preocupação ambiental se espalhou e as organizações encontraram-se sob pressão para cumprir leis e padrões ambientais em suas práticas e adotar uma abordagem sustentável de respeito ao meio ambiente e à sociedade. Nessa linha de pensamento, tem despertado interesse de indivíduos e organizações, ONGs, pesquisadores e outros agentes econômicos; ações

Recebido: 20 Jan., 2020. Aceito: 19 Ago., 2020

E-mail: mqmurad@gmail.com

Este é um artigo publicado em acesso aberto (Open Access) sob a licença Creative Commons Attribution Non-Commercial, que permite uso, distribuição e reprodução em qualquer meio, sem restrições desde que sem fins comerciais e que o trabalho original seja corretamente citado. 
que objetivam desenvolver conceitos de desenvolvimento sustentável e a capacidade de atender às necessidades da geração atual sem comprometer as necessidades das futuras $[3,4]$.

No contexto dos processos de soldagem, relativo à sustentabilidade, o setor sucroalcooleiro se apresenta como um palco para este estudo, pois este segmento possui uma cultura para a inovação com grandes potenciais de atuação em energia renovável, biocombustíveis, bioeletricidade e biomateriais. Como em muitas outras indústrias, este setor enfrenta importantes questões e oportunidades em aspectos relacionados à gestão da sustentabilidade [5].

As moendas, equipamentos destinados ao processo de extração do caldo da cana de açúcar nas empresas sucroalcooleiras, ficam sujeitas, durante a sua operação, a grandes tensões em meios agressivos, em qual ocorrem desgastes acentuados durante o período de moagem [6-8], conforme apresentado nas Figura 1 e 2.
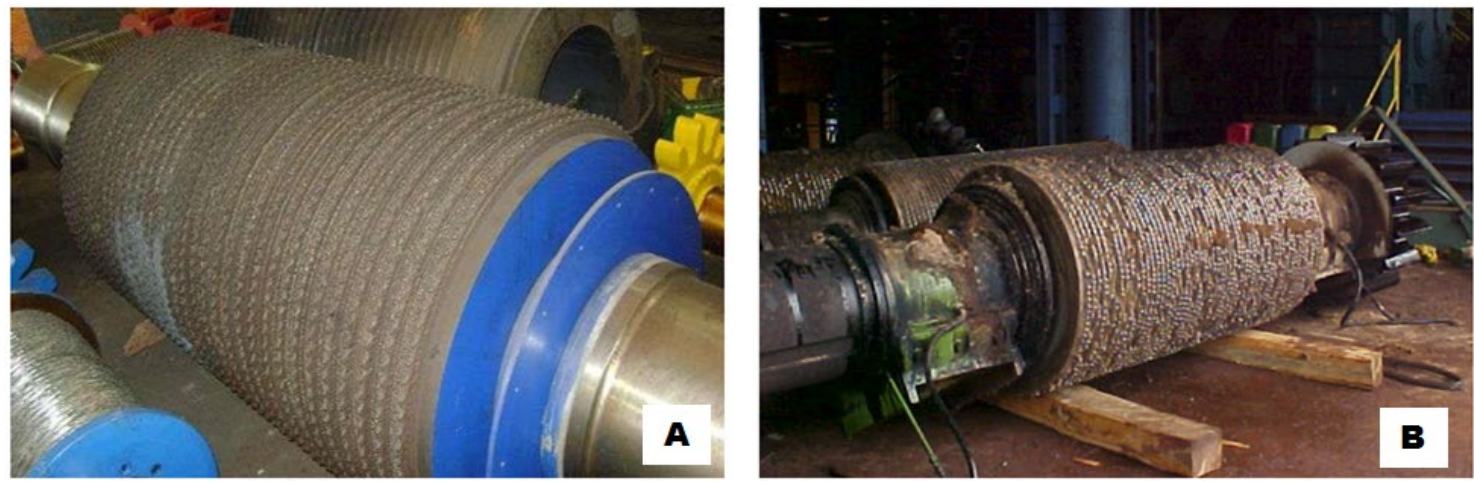

Figura 1. Eixos de moenda antes (A) e depois (B) da operação [9].
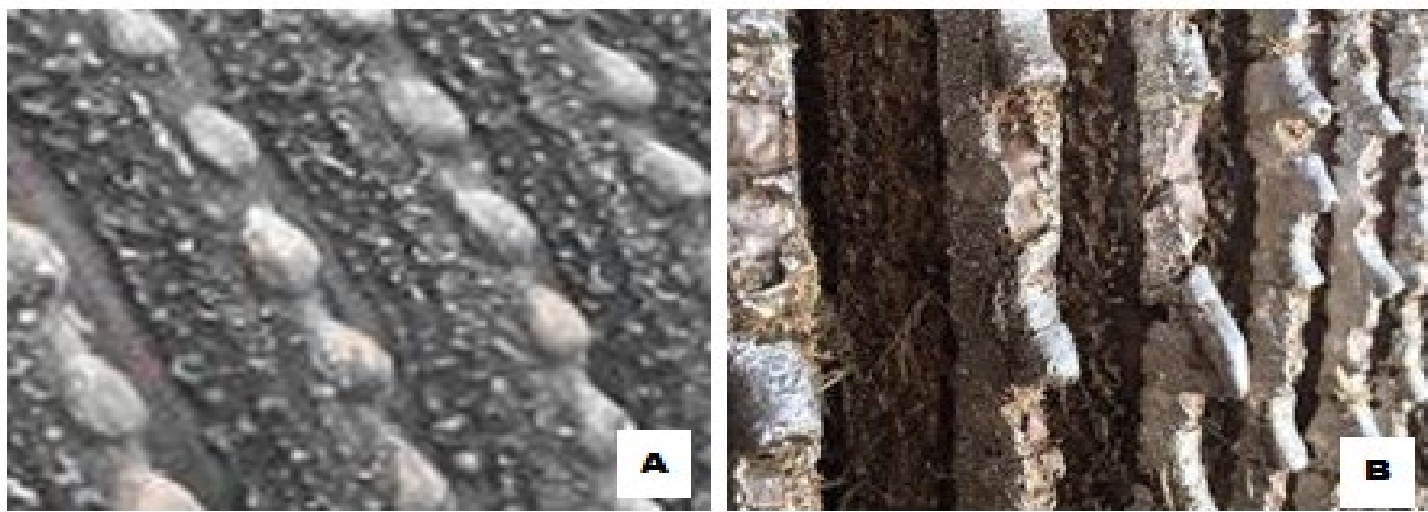

Figura 2. Detalhes dos dentes (ranhuras) de moenda antes (A) e depois (B) da operação.

Para minimizar estes desgastes e aumentar a produção da moenda, nas ranhuras (dentes) da moenda é aplicada uma camada de revestimento duro, como-indicado na Figura 3, podendo utilizar o processo de soldagem Flux-Cored Arc Welding (FCAW) ou o processo Flux-Cored Arc Welding (FCAW) [8-10]. Embora estes depósitos reduzam desgaste, eles também representam um aumento nos custos de manutenção [9]. É usual a aplicação de revestimentos com ligas compostas de cromo, tais componentes são formadores de carbonetos de cromo [11]. É comum a utilização das ligas de Fe-Cr-C [7].

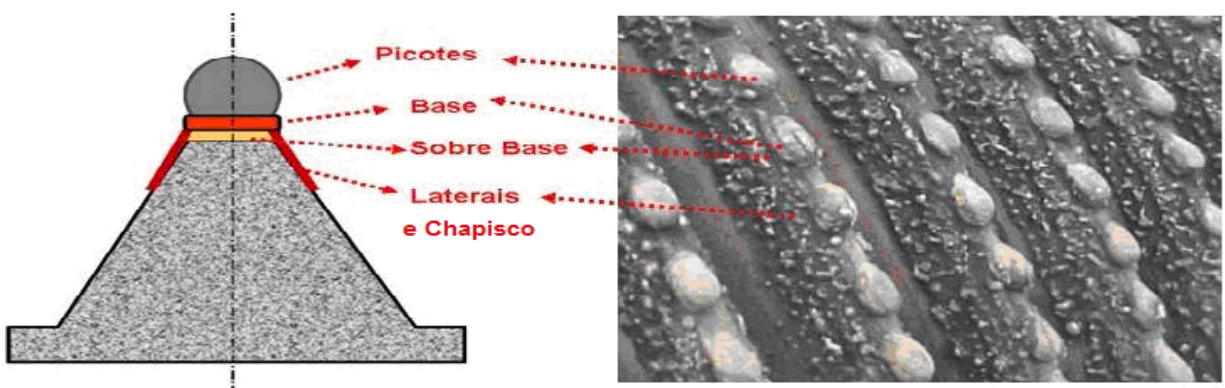

Figura 3. Aplicação de revestimento duro nas ranhuras (dentes) da moenda [9]. 
O chapisco é o revestimento que tem a função de proteger a moenda do desgaste e também prover atrito entre a cana e a moenda, e este atrito é ocasionado pelos grãos formados pelas gotas de metal da solda que se solidificarão devido a presença de água (utilizada junto com a aplicação do chapisco) antes de se fundirem completamente com a poça de fusão

O revestimento duro (chapisco) é aplicado na moenda em duas fases distintas, a primeira na preparação da moenda para a safra, sendo que após o término de uma campanha, as moendas são desmontadas e encaminhadas para a manutenção, e nestes, suas camisas de ferro fundido são submetidas a uma usinagem para retirada do revestimento antigo e uma nova aplicação de revestimento. Este processo é feito de 3 a 5 vezes, enquanto a camisa da moenda permitir a usinagem, sem, contudo, perder as dimensões mínimas necessárias para a sua funcionalidade e resistência do material fundido. Após uma usinagem, é aplicada uma nova camada de revestimento que é realizada de forma automatizada, pelo processo Flux-Cored Arc Welding (FCAW).

Em outra fase, durante a operação do equipamento, é aplicado o revestimento duro nas laterais das ranhuras da moenda. Este processo pode ser realizado com o auxílio um sistema automatizado ou semi-automatizado pelo processo arame tubular (Flux-Cored Arc Welding - FCAW) ou totalmente manual, com o processo eletrodo revestido (Shielded Metal Arc Welding SMAW) [9], como pode ser visto na Figura 4.
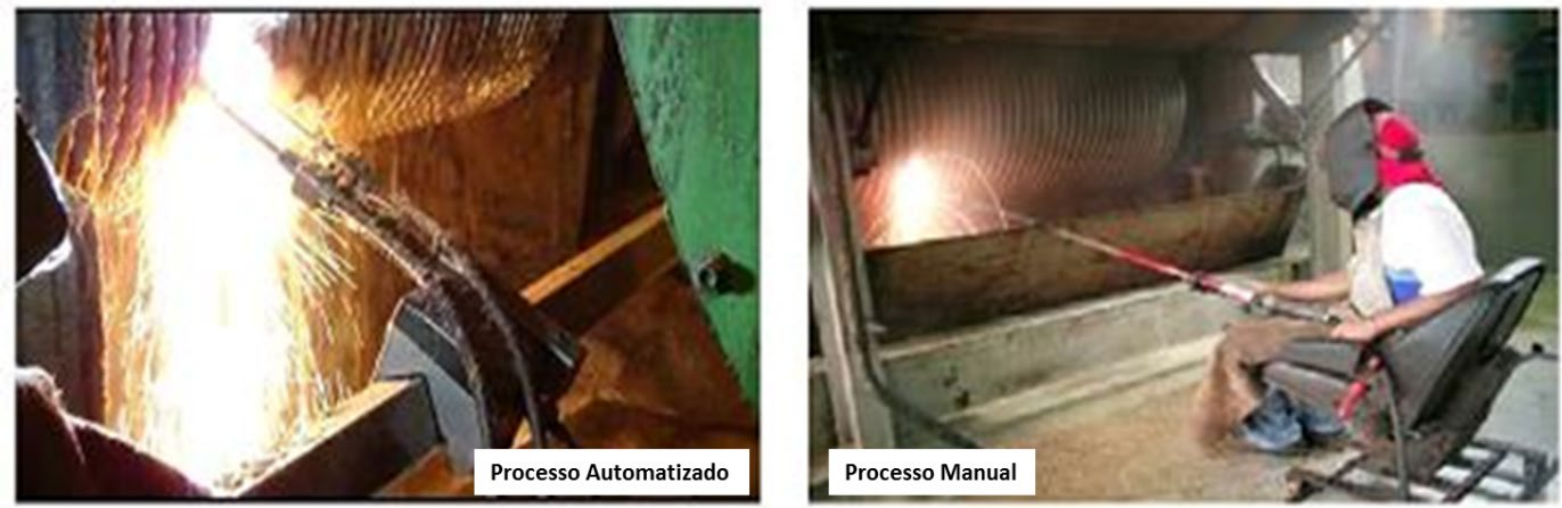

Figura 4. Aplicação automatizada e manual do revestimento duro durante operação [9].

Para Sharma [1] os aspectos de sustentabilidade estão correlacionados a parâmetros de soldagem, entre eles, a taxa de deposição. O processo de soldagem deve depositar tanto quanto o material com o mínimo possível energia elétrica [2]. E também indica em seus estudos, a relação entre a Velocidade de Aplicação de Chapisco (Vc) e a tensão de soldagem, sendo que tais parametrizações resultaria em índice de qualidade distintas. Nascimento e Pukasieiwcz [12] observaram em suas simulações computacionais a ocorrência da redução da diluição com o aumento da velocidade de soldagem e consequentemente ocasionou uma redução no Rendimento de Deposição $(R d)$.

Na mesma linha de pensamento, Sproesser et al. [13] e Chucheep et al. [14] ao estudarem as diferenças entre processos distintos de soldagem, consideraram a Vc como fator decisivo para obter melhores Rd.

O Rendimento de Deposição é definido pela relação do peso inicial do material base com o cordão de solda dividido pelo material base sem o cordão de solda, valores estes em porcentagem [15]. No caso dos eixos de moenda, devido a dificuldade de pesar a moenda (peso estimado de 50 toneladas) o Rd pode ser obtido pela relação entre o peso do arame aplicado durante um determinado tempo e o material que não foi aderido (respingo) durante o processo de soldagem [9].

Santos [16] cita que é esperado um Rd de 50\% para aplicação do revestimento supracitado quando se utiliza o arame tubular, e, para o eletrodo, espera-se $30 \%$. Ou seja, todo material metálico que não agregou no eixo da moenda se tornará um resíduo, implicando em contaminação ambiental e perdas econômicas para a empresa.

Segundo a Norma ABNT NBR 10.004 [17], a classificação dos resíduos sólidos perigosos são aqueles que apresentam periculosidade associada às características de inflamabilidade, corrosividade, reatividade e patogenicidade. Neste contexto, os resíduos gerados pelo processo de soldagem para o revestimento de moendas de cana-de-açúcar são considerados resíduos sólidos perigosos.

Na Ficha de Informações de Segurança de Produtos Químicos (FISPQ), do metal de adição utilizado neste estudo pode-se comprovar a presença de elementos como cromo e níquel na composição do arame tubular auto-protegido [9]. Pela norma ABNTO NBR 10.004/4, o resíduo deste produto é classificado como código F006, ou seja, lodos do tratamento de efluentes líquidos provenientes de operações de eletrodeposição (com presença de cromo hexavalente, níquel e outros), são classificados como produto perigoso.

A gestão da sustentabilidade, que antes tratado unicamente como foco ambiental, quando incorpora o conceito de Triple Bottom Line, considerando impactos sociais e econômicos da empresa, tende a agregar mais valor aos seus bens e serviços, além de fortalecer sua imagem no mercado [4]. Sproesser et al. [13]. e Chucheep et al. [14] consideraram a soldagem sustentável em 
relação às dimensões econômicas e ambientais e realizaram estudos comparando processos distintos de soldagem, apontando as diferenças relativas à sustentabilidade destes processos.

À luz desses propósitos, o objetivo deste artigo foi realizar um estudo onde se alterou o Vc e suas implicações no Rd, e consequentemente os impactos gerados na redução de resíduos e custos, na aplicação de revestimento duro em moendas de cana de açúcar (durante a fase de preparação da moenda para a safra). A proposta foi alterar o valore de Vc e analisar os aspectos de sustentabilidade neste processo de manufatura no setor sucroalcooleiro, em específico na geração de resíduos provenientes da aplicação de revestimento metálico pelo processo de soldagem por arame tubular (FCAW) e os aspectos econômicos relacionados ao processo. Assim, foram conduzidos estudos em duas linhas distintas: a) Econômica e Tecnológica, focando na melhoria da aplicação, mediante alteração de parâmetros de soldagem; b) Ambiental: com foco na geração de resíduos e eficiência energética.

\section{Metodologia}

Os ensaios foram realizados em uma empresa localizada no interior do estado de São Paulo que atualmente é referência no mercado sucroalcooleiro em venda de produtos fundidos e usinados. A empresa permitiu o acesso à sua planta e, em específico, ao departamento de soldagem onde foram realizados os ensaios deste estudo, além de fornecer os insumos necessários para este experimento bem como o acompanhamento de técnicos e supervisores.

A Figura 5, que mostra o dispositivo de soldagem empregado para a aplicação do revestimento duro nas camisas de moenda. Nesta, pode-se visualizar o sistema de alimentação de água, o equipamento de soldagem e o dispositivo mecânico que confere a rotação da camisa de moenda que receberá a aplicação de revestimento duro.

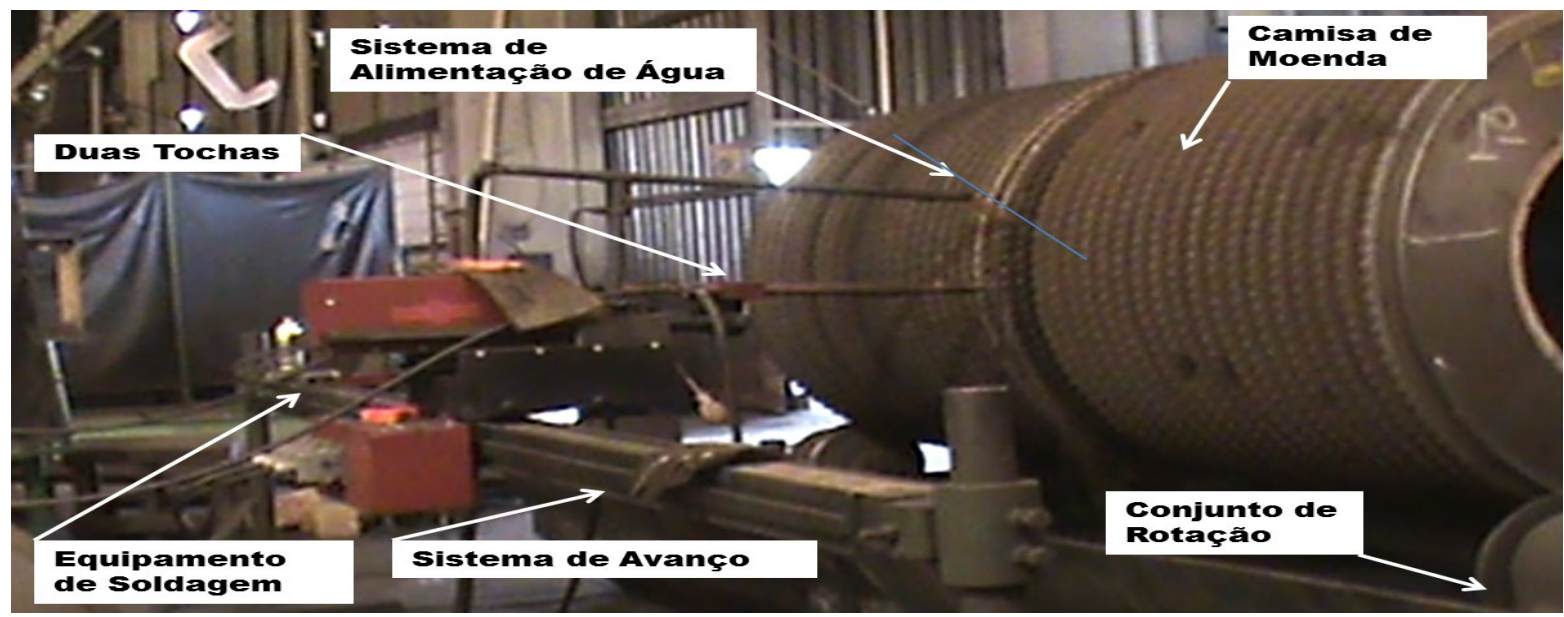

Figura 5. Conjunto utilizado para realização da aplicação do revestimento [9].

A camisa de moenda é montada na posição horizontal em um dispositivo mecânico que lhe confere uma rotação que, por sua vez, pode ser variada por intermédio de um inversor de frequência. Conforme os procedimentos operacionais adotados pela empresa, a Vc pode ser variada entre 2 a $20 \mathrm{~m} / \mathrm{min}$, sendo que a mais utilizada é a de $10 \mathrm{~m} / \mathrm{min}$. A camisa deve ser apoiada sobre roletes ou mancal de forma que não haja deslizamento durante seu trabalho, garantindo um perfeito alinhamento ao dispositivo de aplicação do revestimento duro.

Para a realização da aplicação do revestimento nas laterais dos dentes da moenda é utilizado um dispositivo mecânico acoplado a um conjunto de equipamento de soldagem, constituído de:

a) Fonte de soldagem com capacidade de $600 \mathrm{~A}$ à $100 \%$ de ciclo de trabalho;

b) Cabeçote alimentador com roldanas com canal recartilhado para trabalhar com arames tubulares de até 2,8 milímetros de diâmetro;

c) Unidade de Processamento - PLC, que aciona e comanda os motores dos eixos de avanço e posicionamento. Tais movimentos são controlados por sensores de posição montados no equipamento. Uma unidade Interface Homem Máquina (IHM), permite os ajustes necessários de variação de programação que pode ser realizada pelo operador do equipamento de soldagem.

O metal de base utilizado foi ferro fundido nodular Tipo GGG-60, conforme norma DIN 1693.

O metal de adição foi um arame tubular auto-protegido, com dureza de 57 a 62 HRc, diâmetro de 2,8 mm. A Tabela 1 apresenta a composição química do metal de adição fornecida pelo fabricante. É um produto especialmente projetado para aplicação de revestimento duro em camisas de moenda. 
Tabela 1. Composição química do metal de adição para o revestimento duro.

\begin{tabular}{cccccccc}
\hline $\mathbf{C}$ & $\mathbf{C r}$ & $\mathbf{S i}$ & $\mathbf{V}$ & $\mathbf{M n}$ & $\mathbf{F e}$ & $\mathbf{S}$ & $\mathbf{P}$ \\
$3,64 \%$ & $16,81 \%$ & $1,05 \%$ & $0,50 \%$ & $0,82 \%$ & $76,61 \%$ & $0,01 \%$ & $0,02 \%$ \\
\hline
\end{tabular}

Fonte: Murad [9]

O processo mais usual para obtenção do Rd, relação entre o metal fundido e o que foi efetivamente depositado, é realizado pela pesagem do metal antes e após a aplicação do revestimento. Devido a dificuldade de pesar a moenda (peso estimado de 50 toneladas) o Rd foi obtido neste trabalho pela relação entre o peso do arame aplicado e o material respingado (metal que não foi aderido a moenda). O valor obtido não representa a realidade em termos de Rd (fusão do fluxo do arame tubular), mas é possível obter um valor que possa ser comparado com outros que utilizam a mesma técnica. Para obter o peso do material respingado, foram utilizados os seguintes procedimentos: i) Utilizou-se um recipiente de coleta metálico de aproximadamente 60 litros de volume, dotado de um orifício para a drenagem da água; ii) O recipiente de coleta foi inserido abaixo das tochas e do metal base (camisa da moenda), por um período de tempo de um minuto (tempo de ensaio em campo). Realizado três vezes para cada amostra; iii) Utilizando uma peneira metálica, fez-se a primeira separação do material sólido do líquido; iv) O material foi secado em uma estufa e posteriormente pesado em uma balança analítica com capacidade de 4200 g e leitura de 0,01 g;

Para se obter o peso do material aplicado (metal de adição) foi utilizado o seguinte procedimento: i) Foi coletada (por um minuto) a quantidade de arame que a fonte fornece durante a aplicação do revestimento duro; ii) Posteriormente, foi pesado em uma balança analítica com capacidade de $4200 \mathrm{~g}$ e leitura de $0,01 \mathrm{~g}$; iii) Como o processo utiliza de duas tochas para aplicação do revestimento duro nas mesmas condições de aplicação, o valor obtido foi dividido por 2, para obter os valores do material respingado; iv) Este procedimento foi repetido para as 3 coletas.

A partir das condições utilizadas pela empresa, na qual a mesma adota velocidade $\mathrm{Vc}=10 \mathrm{~m} / \mathrm{min}$, variou-se $\mathrm{o}$ Vc ( 6 e $13,6 \mathrm{~m} / \mathrm{min}$ ) com o objetivo de entender e encontrar novos parâmetros com melhor condição de aplicação, isto é, melhor Rd, e estes valores são apresentados na Tabela 2.

Tabela 2. Parâmetros utilizados nos ensaios em campo.

\begin{tabular}{ccccccc}
\hline Vc & Ud (V) & Va & Aplicação & DPP & Ac & Sentido \\
6 & 31 & 3,2 & Água & 24 & $35^{\circ}$ & Horário \\
10 & 31 & 3,2 & Água & 24 & $35^{\circ}$ & Horário \\
13,6 & 31 & 3,2 & Água & 24 & $35^{\circ}$ & Horário \\
\hline
\end{tabular}

Legenda: Ud tensão desejada (V), Aplicação a seco ou com água; DDP: Distância Bico Contato peça (mm); Va: velocidades de alimentação do arame eletrodo (m/min); Ac: Ângulo de Aplicação (graus) e sentido horário (H) ou anti-horário (AH).

Com o objetivo de obter os valores de corrente e tensão dos ensaios realizados durante a fase de experimento em campo, foi instalado um equipamento com capacidade de registro de $10 \mathrm{kHz}$ para posterior análise. $\mathrm{O}$ equipamento utilizado foi um ADS1800 da marca Lynx.

\section{Resultados e Discussões}

Com as condições de aplicação do revestimento duro empregadas pela empresa e com variação na Vc, foram também obtidos os valores referentes ao peso do material depositado, como massa do Arame Eletrodo (Al) e do Respingo (Resp). O respingo refere-se ao material que não aderiu ao friso da moenda, coletado durante a realização do ensaio para a determinação do Rd.

Os primeiros ensaios realizados em campo foram com os parâmetros de aplicação de revestimento duro da empresa (ensaios 4, 5 e 6), e estão apresentados na Tabela 3. Os outros ensaios com Vc de 6 e 13,6 m/min, apresentados na mesma tabela, foram propostos pela pesquisa com o objetivo de entender o efeito desta variável no aspecto do revestimento duro e principalmente no Rd.

Foram obtidos para cada condição de aplicação, medidas de Im e Um correspondentes à média das amostras obtidas nos ensaios através do sistema de aquisição de dados. As condições de aplicações mantidas constantes, conforme já apresentado na Tabela 3. O tempo de realização do ensaio foi de 1 minuto, houve aplicação de água no arco elétrico. 
Tabela 3. Resultados obtidos com variação de Vc.

\begin{tabular}{|c|c|c|c|c|c|c|c|c|}
\hline \multirow{2}{*}{ Amostra } & Ud & Va & Um & Im & Vc & Al & Resp & Rd \\
\hline & (V) & (m/min) & (V) & (A) & $(\mathrm{m} / \mathrm{min})$ & (g) & (g) & (\%) \\
\hline 1 & 31 & 3,2 & & & 6 & 188,0 & 61,69 & 67,19 \\
\hline 2 & 31 & 3,2 & 36,4 & 313,1 & 6 & 188,0 & 64,50 & 65,69 \\
\hline \multirow[t]{2}{*}{3} & 31 & 3,2 & & & 6 & 188,0 & 66,73 & 64,51 \\
\hline & & Média de Rd & 65,69 & & \multicolumn{3}{|c|}{ Desvio Padrão de Rd } & 1,34 \\
\hline 4 & 31 & 3,2 & & & 10 & 188,0 & 85,28 & 54,64 \\
\hline 5 & 31 & 3,2 & 37,1 & 309,0 & 10 & 188,0 & 81,08 & 56,87 \\
\hline \multirow[t]{2}{*}{6} & 31 & 3,2 & & & 10 & 188,0 & 85,50 & 54,52 \\
\hline & & Média de Rd & 54,64 & & \multicolumn{3}{|c|}{ Desvio Padrão de Rd } & 1,32 \\
\hline 7 & 31 & 3,2 & & & 13,6 & 188,0 & 102,40 & 45,53 \\
\hline 8 & 31 & 3,2 & 36,77 & 297,5 & 13,6 & 188,0 & 102,80 & 45,32 \\
\hline \multirow[t]{2}{*}{9} & 31 & 3,2 & & & 13,6 & 188,0 & 102,00 & 45,74 \\
\hline & & Média de Rd & 45,53 & & \multicolumn{3}{|c|}{ Desvio Padrão de Rd } & 0,21 \\
\hline
\end{tabular}

Legenda: Ud: Tensão desejada; Um: Tensão média; Im: Corrente média; Resp: Respingo; Al: Massa de arame eletrodo a ser fundida em 1 minuto de ensaio.

Verifica-se na Tabela 3 que existe uma diferença entre os valores desejados Ud e os valores médios de tensão (Um) obtidos pelo sistema de aquisição. Por ser um equipamento construído de forma dedicada à aplicação de revestimento duro, não é possível alterar os valores no equipamento, pois ficaria diferente daqueles realizados pela empresa.

Foi realizada análise estatística do $\mathrm{Rd}$ em função da Vc, com definição de um nível de significância de $5 \%$, isto é, uma confiabilidade de $95 \%$. Todas caracterizando-se como estatisticamente diferentes, com confiabilidade de $95 \%$.

A Figura 6 apresenta os valores do Rd em função da Vc. Verifica que o aumento da Vc diminui significativamente o Rd, fato este semelhantes aos observados nos estudos de Sproesser et al. [13] e Chucheep et al. [14] que consideraram a Vc como fator decisivo para obter melhores Rendimento de Deposição (Rd). Nota-se que houve uma redução de $20,22 \%$ quando se elevou a Vc de 6 para $10 \mathrm{~m} / \mathrm{min}$, e uma redução de 20,08\% quando se elevou de 10 para 13,6 m/min. As reduções de Rd se justificam pela redução da diluição e dificuldade de se formar poça de fusão em Vc maiores, como ocorre na soldagem de um cordão de solda, fato apresentado nas simulações computacionais de Nascimento e Pukasieiwcz [12]. O objetivo aqui é obter pequenas gotas de metal depositado ao longo do friso da moenda. Entretanto, a maior Vc aumenta a dificuldade de aderência das gotas no friso da moenda, gerando uma maior quantidade de respingo e, como consequência, a diminuição do Rd. Tais fatos evidenciados também foram observados por Sharma [1], em que os aspectos de qualidade e consequentemente de sustentabilidade são afetados pelos Rd e estes foram influenciados pela Vc.

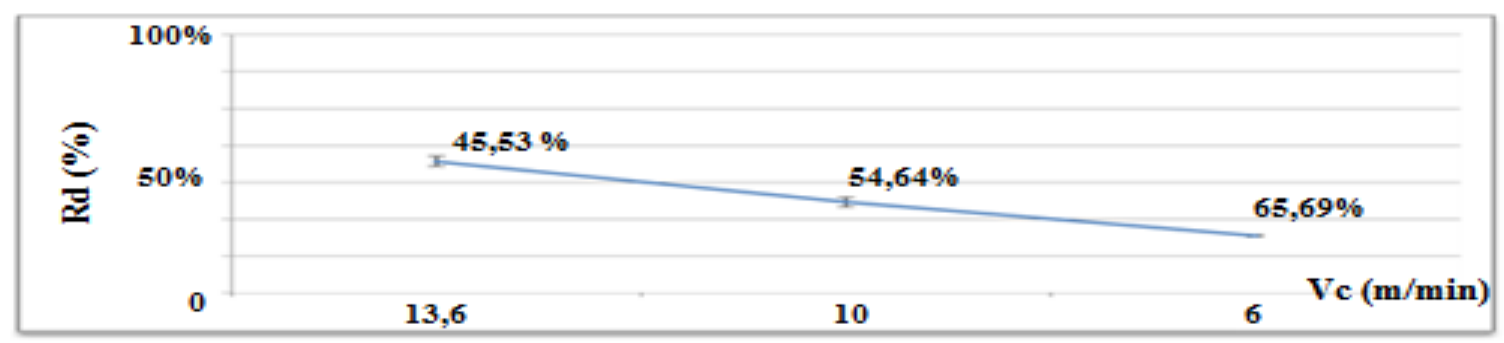

Figura 6. Resultados obtidos com a variação de Vc.

As Figuras 7 a 9 apresentam os oscilogramas de tensão e corrente para cada condição de Vc realizado em campo. Verificase que os valores de corrente medida $(\mathrm{Im})$ sofrem uma redução com a elevação de Vc. Observa-se também que a amplitude pico a pico aumentam com a elevação de Vc. Estes fatos fazem com que a geração de respingo aumente, diminuindo o Rd. Novos estudos deverão ser realizados no futuro para explicar tais fatos para esta aplicação, pois estudos de outros pesquisadores como Wang et al. [2] demonstraram comportamento distintos. Tal evidenciação é semelhante à observada por Sharma [1] sendo que, além desta, ficou evidenciado que este fator também influencia resultados de sustentabilidade. 

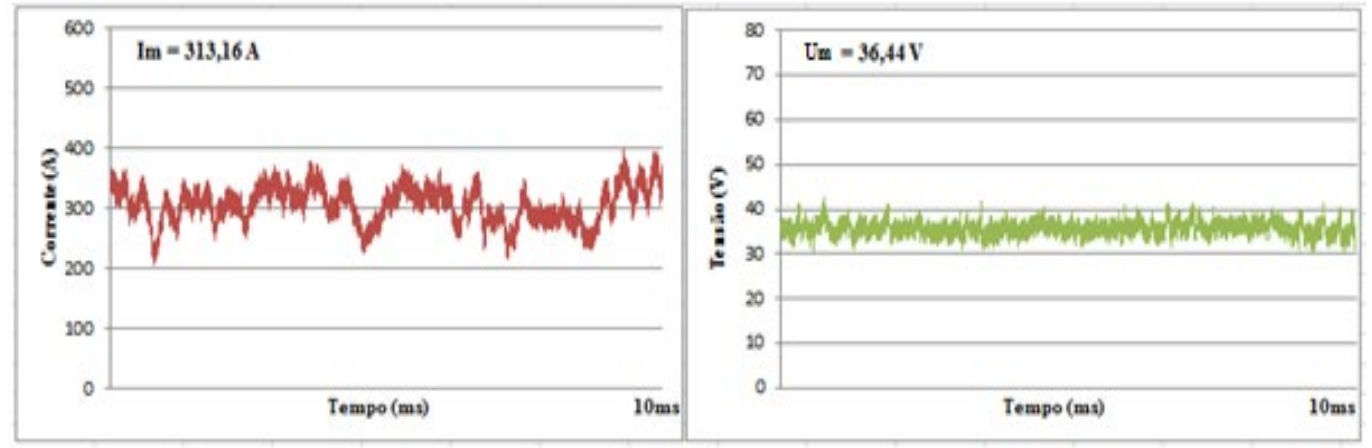

Figura 7. Oscilogramas de tensão e corrente para $6 \mathrm{~m} / \mathrm{min}$ e $300 \mathrm{~A}$.
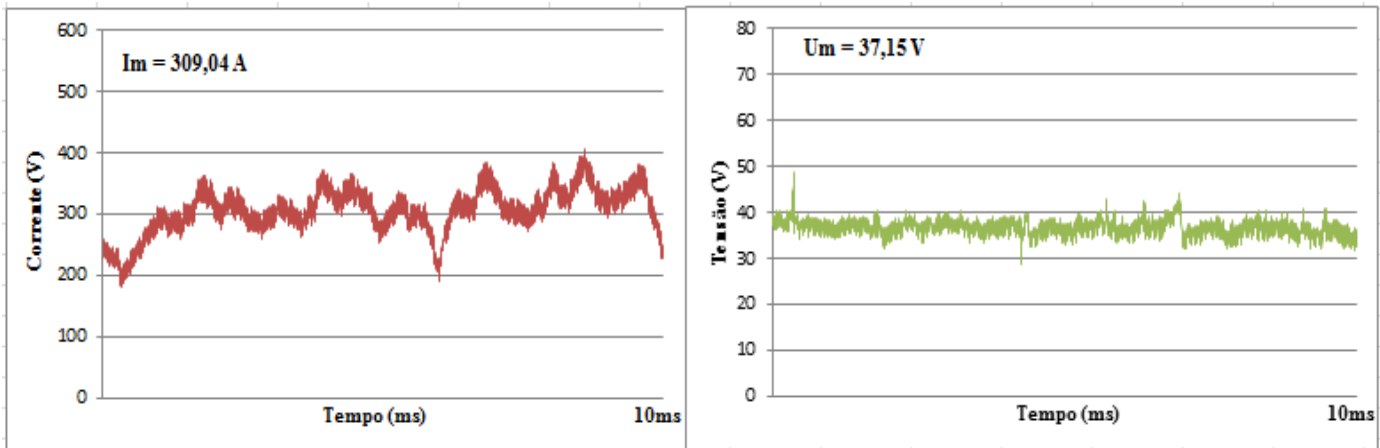

Figura 8. Oscilogramas de corrente e tensão para $10 \mathrm{~m} / \mathrm{min}$ e $300 \mathrm{~A}$.
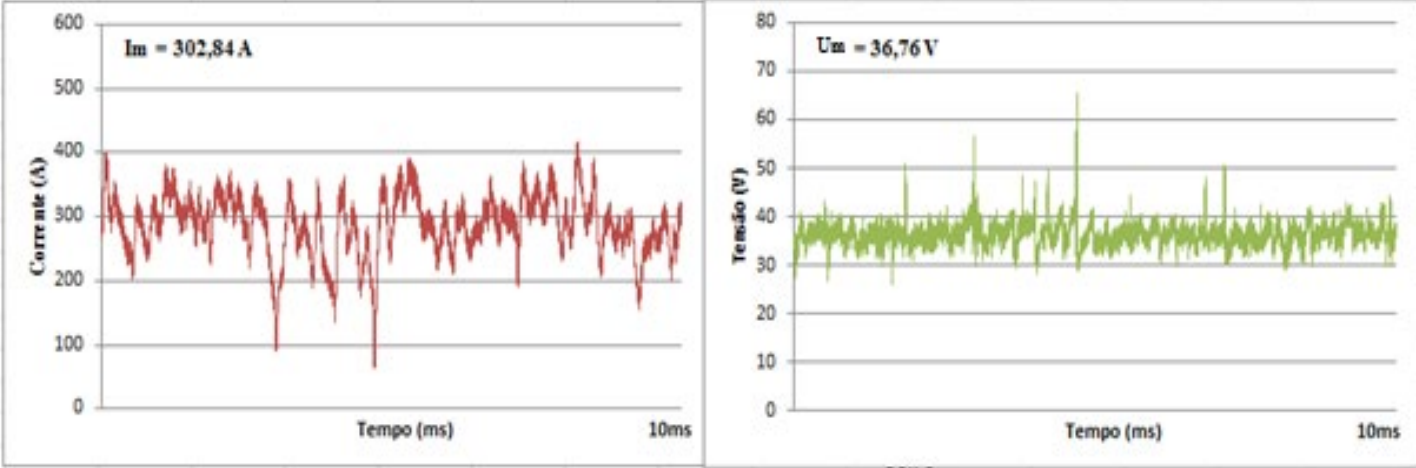

Figura 9. Oscilogramas de corrente e tensão para $13,6 \mathrm{~m} / \mathrm{min}$ e $300 \mathrm{~A}$.

Na Figura 10 são apresentados os aspectos dos revestimentos duros aplicados no friso da moenda (dentes) para cada uma das condições de Vc, na qual é possível visualizar as alterações nas quantidades de grão e nos tamanhos de grão. Nota-se que nas menores $\mathrm{Vc}$ apresentaram melhores $\mathrm{Rd}$, e também apresentaram grãos de maior dimensão e em maiores quantidades, características estas desejada para esta aplicação. Na Figura 11 é apresentada a contagem da quantidade de grão em uma área de $4 \mathrm{~cm}^{2}$, e nesta duas amostras aprovadas.

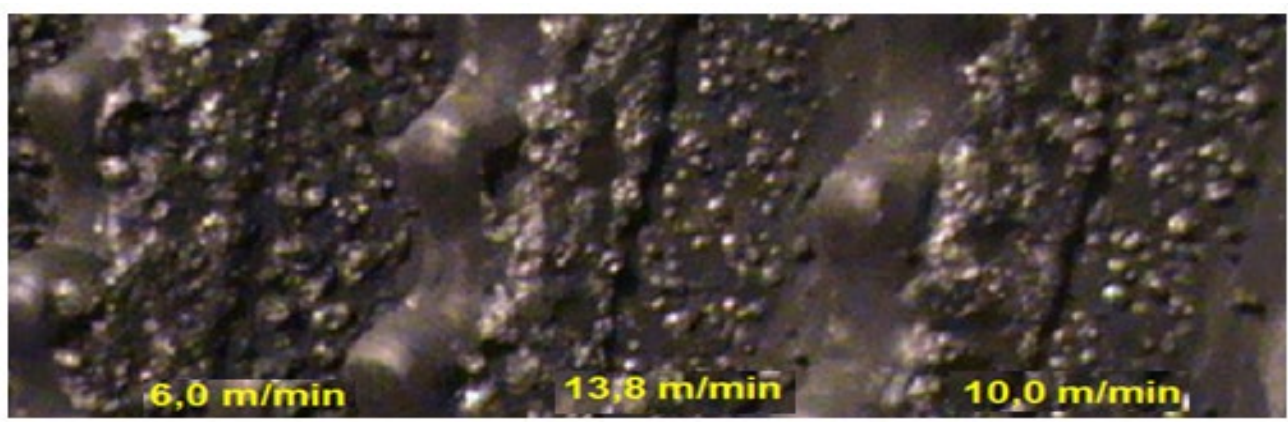

Figura 10. Aspecto do revestimento duro obtido com a variação da Vc. 


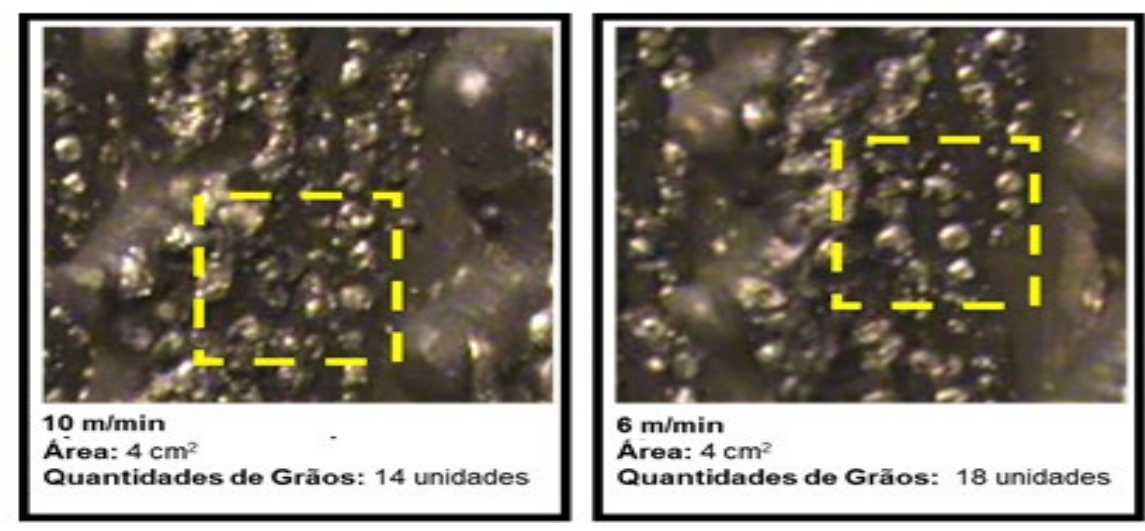

Figura 11. Quantidade de grão obtidos para 6 e $10 \mathrm{~m} / \mathrm{min}$.

Não existe um padrão oficial adotados pelas empresas para o controle de qualidade deste revestimento. Assim, o critério adotado para a aprovação ou reprovação deste revestimento é apenas o visual. Neste contexto foi solicitado ao engenheiro responsável do setor de aplicação do chapisco que fizesse a sua avaliação. Nesta análise foram aprovados os ensaios com 6 e $10 \mathrm{~m} / \mathrm{min}$ e reprovada a aplicação com 13,8 m/min, tendo como motivo a pequena quantidade de material aderido aos dentes da moenda.

Verifica-se na Figura 10, para a Vc em 13,5 m/min, que os aspectos de qualidade do revestimento duro obtido não foram satisfatórios, pois os grãos sofreram uma redução significativa em seu diâmetro. Este fato demonstra que o aumento da Vc implica em uma queda na qualidade desejada, obtendo-se uma quantidade grande de grãos com dimensões inferiores aos desejado pela empresa. Este resultado implicou também em uma queda no valor do Rd.

Verifica-se, mediante tais resultados, que o emprego de Vc de $6 \mathrm{~m} / \mathrm{min}$ obtém um Rd de 65,69\% contra 54,64\% daquele empregado pelas empresas fabricantes deste equipamento ( $\mathrm{Vc}=10 \mathrm{~m} / \mathrm{min}$ ), ou seja, um ganho de $20,22 \%$ na quantidade de material depositado, mantendo praticamente a mesma qualidade de aplicação do revestimento duro. Entretanto, existe uma maior demora na aplicação, isto é, uma menor produtividade. É necessária uma análise de custo para poder justificar a necessidade de uma diminuição na Vc. Tal evidenciação é semelhante a observada por Sharma [1], sendo que esta pesquisa adiciona que a influência no aspecto da qualidade impacta também a dimensão da sustentabilidade.

Analisou-se, na sequência, uma comparação de custo, levando em conta os custos relativos a mão de obra do operador de máquina (salário médio de $\mathrm{R} \$ 2.500$, ou seja, um custo de $\mathrm{R} \$ 23,00 /$ hora). Não foram considerados os valores referentes aos custos de máquinas e equipamentos, pois uma análise específica apenas seria possível a partir dos dados de tempos de máquina e tempos, valores de depreciação e outros valores específicos segundo as particularidades de cada empresa.

Considerando que houve um ganho de $\mathrm{Rd}$ de $20,22 \%$ (ou seja $65,69 \%$ obtidos neste estudo comparado aos $54,64 \%$ praticados pela empresa), seria prudente afirmar que poderia haver redução na quantidade de passes para se aplicar a mesma quantidade de material. Dessa forma, foi considerado um fator de correção (FP) de 20,22\% no valor final.

Para os cálculos de custos relativos a aplicação de uma moenda com dimensões $1100 \mathrm{~mm}$ de diâmetro por $2200 \mathrm{~mm}$ de comprimento contendo 57 frisos, são necessários 5 horas, como tempo de execução (TE) para aplicação do revestimento, aplicando-se de 10 a 12 passes por friso, com Vc de $10 \mathrm{~m} / \mathrm{min}$ e 8 horas para Vc de $6 \mathrm{~m} / \mathrm{min}$. Os custos de matéria-prima (MP) foram apresentados conforme o rendimento, ou seja 60 quilogramas para aplicação com $10 \mathrm{~m} / \mathrm{min}(\mathrm{Rd}=54,64 \%)$ e $26,68 \mathrm{Kg}$ para $6 \mathrm{~m} / \mathrm{mim}(\mathrm{Rd}=65,69 \%)$. E, por fim, foi considerado o valor de $\mathrm{R} \$ 15,00$ por quilo de arame, como custo de matéria-prima (MP). A Tabela 4 apresenta estes custos referentes a um rolo de moenda.

Tabela 4. Custo de aplicação de revestimento duro para um rolo de moenda.

\begin{tabular}{|c|c|c|c|c|c|c|c|c|}
\hline $\mathrm{Vc}(\mathrm{m} / \mathrm{min})$ & TE (horas) & $\begin{array}{c}\text { Custo M.O. } \\
\text { (R\$) }\end{array}$ & $\operatorname{Rd}(\%)$ & MP (Kg) & FP (R\$) & $\begin{array}{l}\text { Custo } \\
\text { MP (R\$) }\end{array}$ & $\begin{array}{l}\text { Custo Final } \\
\text { (R\$) }\end{array}$ & $\begin{array}{l}\text { Custo Final } \\
\text { com FP (R\$) }\end{array}$ \\
\hline 10 & 5 & 115 & 54,64 & 60 & 0 & 900 & 1015 & 1015 \\
\hline 6 & 8 & 161 & 65,69 & 53,38 & 192,2 & 800,04 & 961,04 & 768,84 \\
\hline \multicolumn{9}{|c|}{ Economia por Rolo de Moenda de R\$ 246,16 (Redução de 32\%) } \\
\hline
\end{tabular}

Legenda: TE: tempo de execução; M.O.: mão de obra; MP: matéria prima; FP: fator de correção.

A empresa parceira realiza este trabalho em cerca de aproximadamente 1000 moendas por ano, o custo reduzido seria de R\$ 246.160,00 por ano. Considerando também que são utilizados aproximadamente 4.000 rolos de moenda no Brasil, esta economia alcançaria o valor $\mathrm{R} \$ 984.640,00$ por ano. 
Se o mesmo raciocínio fosse estendido para a manutenção moenda em safra (aplicação de revestimento durante a operação) e considerarmos que são vendidos 3,5 milhões de Kg de material de adição (valor fornecido pelo fabricante do

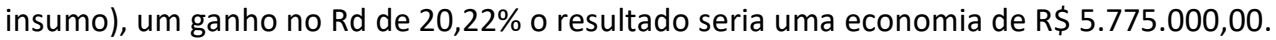

Por fim, o ganho estimado para a preparação da moenda e manutenção em safra seria de aproximadamente R\$ 6.759.640,00 no Brasil.

Tal evidenciação é corroborada por Hill e Seabrook [4], os quais enfatizam a gestão da sustentabilidade sob o paradigma do Triple Bottom Line. Os aspectos econômicos e ambientais obtidos com a redução da Vc contribuem de forma significativa no que diz respeito aos parâmetros de sustentabilidade.

É fato que a sustentabilidade na produção não se resume apenas à geração de resíduos, eficiência energética e custo, aspectos estes relacionados ao Triple Bottom Line, mas estes aspectos contribuem para uma manufatura mais sustentável, corroborando com os estudos de Solomon et al. [5], Sproesser et al. [13]. e Chucheep et al. [14].

No entanto, este estudo realizou uma análise considerando apenas estes três temas. Sobre a geração de resíduo, deve-se considerar aqui que o respingo do revestimento duro, tem como destino o decantador do caldo da cana, equipamento este que realiza a separação de rejeitos sólidos e este rejeito (torta do filtro) tem como destino final a lavoura de cana como forma de adubação. Deve-se considerar também que este respingo é considerado um sólido perigoso, segundo a Norma ABNT NBR 10.004 [14], que trata da classificação de resíduos sólidos perigosos.

Partindo-se do exposto de que são vendidos 3,5 milhões de kg de metal de adição para realização de revestimento duro. E considerando que todo material de adição não aderido (material do respingo se torna um resíduo que provoca impactos ambientais. Pode-se afirmar que um ganho de $20,22 \%$ no Rd reduziria em $385.000 \mathrm{Kg}$ a geração de resíduos perigosos por ano.

Em relação ao ganho energético, seguindo o mesmo raciocínio anterior, de forma simplificada pode-se afirmar que um ganho de $20,22 \%$ no $\mathrm{Rd}$, resultaria em um ganho na eficiência energética.

\section{Conclusão}

A realização deste trabalho permitiu chegar às seguintes conclusões:

- A redução da velocidade de aplicação de chapisco durante a preparação da moenda acarretou em um ganho de $20,22 \%$ de rendimento de deposição, isto é, redução da quantidade de material desperdiçado;

A menor velocidade de aplicação do chapisco implicou também na diminuição do custo final de preparação da moenda para a safra de aproximadamente $32 \%$. Isto ocorreu devido a melhor eficiência de deposição;

- A melhor condição de aplicação do chapisco (menor Vc) obtida neste trabalho induz uma redução na geração de 385.000 $\mathrm{Kg}$ (material do respingo) de resíduos perigosos por ano.

Por fim considerando os pilares econômicos e ambientais do Triple Bottom Line, a redução dos custos de aplicação, do consumo de energia elétrica e a geração sem comprometer a qualidade final do revestimento duro contribuem de forma significativa para a sustentabilidade na produção.

\section{Agradecimentos}

Os autores expressam o seu agradecimento às instituições que apoiaram para realização deste trabalho: Cefores/UFTM e UFU.

\section{Referências}

[1] Sharma A. A fundamental study on qualitatively viable sustainable welding process maps. Journal of Manufacturing Systems. 2018;46:221-230. http://dx.doi.org/10.1016/j.jmsy.2018.01.002.

[2] Wang J, Sun Q, Pan Z, Yang J, Feng J. Effects of welding speed on bubble dynamics and process stability in mechanical constraint-assisted underwater wet welding of steel sheets. Journal of Materials Processing Technology. 2019;264:389-401. http://dx.doi.org/10.1016/j.jmatprotec.2018.09.022.

[3] Cezarino LO, Murad QM, Rezende PV, Falco Sales W. Being green makes me greener? An evaluation of sustainability rebound effects. Journal of Cleaner Production. 2020;269:121436. http://dx.doi.org/10.1016/j.jclepro.2020.121436.

[4] Hill DC, Seabrook KA. Safety \& sustainability: understanding the business value. Park Ridge, IL: American Society of Safety Engineers, 2013.

[5] Solomon S, Quirk RG, Shukla SK. Special issue: green management for sustainable sugar industry. Sugar Tech. 2019;21(2):183-185. http://dx.doi.org/10.1007/s12355-019-00711-2.

[6] Buchanan VE, Shipway PH, McCartney DG. Microstructure and abrasive wear behaviour of shielded metal arc welding hardfacings used in the sugarcane industry. Wear. 2007;263(1-6):99-110. http://dx.doi.org/10.1016/j.wear.2006.12.053. 
[7] Casanova F, Aguilar Y. A study on the wear of sugar cane rolls. Wear. 2008;265(1-2):236-243. http://dx.doi.org/10.1016/j.wear.2007.10.008.

[8] Rivas JS, Coronado JJ, Gómez AL. Tribological aspects for the shafts and bearings of sugar cane mills. Wear. 2006;261(7-8):779-784. http://dx.doi.org/10.1016/j.wear.2006.01.026.

[9] Murad MQ. Aplicação de chapisco em moenda de cana de açúcar com o processo FCWA [dissertação de mestrado]. Uberlândia: Universidade Federal de Uberlândia; 2015.

[10] Hugot E. Handbook of cane sugar engineering. 3rd ed. New York: Elsevier; 1986.

[11] Lima A, Ferraresi V. Análise da microestrutura e da resistência ao desgaste de revestimento duro utilizado pela indústria sucroalcooleira Soldagem e Inspeção. 2009;14(2):140-150. http://dx.doi.org/10.1590/S0104-92242009000200006.

[12] Nascimento G, Pukasieiwcz AGM. Análise do processo de deposição FCAW de uma liga de carboneto de cromo aplicando o planejamento experimental central composite circumscribed. Soldagem e Inspeção. 2017;21(4):444-455. http://dx.doi.org/10.1590/0104-9224/si2104.05.

[13] Sproesser G, Schenker S, Pittner A, Borndörfer R, Rethmeier M, Chang Y-J, et al. Sustainable welding process selection based on weight space partitions. Procedia CIRP. 2016;40:127-132. http://dx.doi.org/10.1016/j.procir.2016.01.077.

[14] Chucheep T, Thangwichien N, Mahathaninwong N, Janudom S, Yirong C. Welding quality and sustainability of alternative LPG valve boss welding processes. Songklanakarin Journal of Science and Technology. 2019;41(5):1146-1153.

[15] Thakur A, Gebrelibanos H, Gabrey T. Arc welding process selection through a quality and costs. International Journal of Current Engineering and Technology. 2019;9(3). http://dx.doi.org/10.14741/ijcet/v.9.3.6.

[16] Santos SA. Revestimentos automatizados em camisas de moendas. Boehler Thyssen; 2001. (Boletim UTP).

[17] Associação Brasileira de Normas Técnicas. NBR 10004: resíduos sólidos: classificação. Rio de Janeiro: ABNT; 2004. p. 9-11. 